
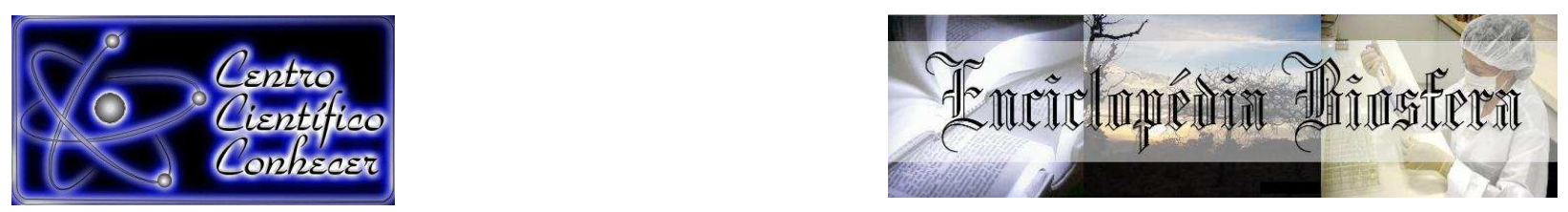

\title{
AVALIAÇÃO ULTRASSONOGRÁFICA DOS LINFONODOS AXILARES E INGUINAIS EM CADELA COM TUMOR MAMÁRIO: RELATO DE CASO
}

\section{Rejane Guerra Ribeiro ${ }^{1}$, Carla Amorim Neves ${ }^{2}$, Tales Dias do Prado ${ }^{3}$, Naida Cristina Borges $^{4}$, Andrigo Barboza De Nardi ${ }^{5}$}

1 Doutorando (a) do Programa de Pós-Graduação em Medicina Veterinária da Universidade Estadual Paulista - UNESP (rejane.guerra.vet@hotmail.com) Câmpus de Jaboticabal - SP-Brasil

2 Residente do Setor de Diagnóstico por Imagem do Hospital Veterinário da Universidade Federal de Goiás- UFG

3 Professsor Doutor do Departamento de Clínica e Cirurgia Veterinária da Universidade de Rio Verde- UniRV

4 Professora Doutora do Departamento de Diagnóstico por Imagem da Universidade

Federal de Goiás- UFG

5 Professor Doutor do Departamento de Clínica e Cirurgia Veterinária da Universidade Estadual Paulista-UNESP, Câmpus de Jaboticabal

\section{Recebido em: 08/04/2016 - Aprovado em: 30/05/2016 - Publicado em: 20/06/2016} DOI: 10.18677/Enciclopedia_Biosfera_2016_020

\section{RESUMO}

O estudo das neoplasias mamárias é relevante em função da alta casuística e impacto na sobrevida do animal. A avaliação dos linfonodos faz parte do estadiamento das neoplasias mamárias, e é um importante indicador de prognóstico. O objetivo deste trabalho é ressaltar a importância da avaliação ultrassonográfica dos linfonodos axilar e inguinal na pesquisa de metástase.

PALAVRAS-CHAVE: canino, linfonodos, metástase, tumor

\section{AXILLARY AND INGUINAL LYMPH NODES ULTRASOUND EVALUATION IN A BITCH WITH BREAST TUMOR}

\begin{abstract}
The study of mammary tumors is relevant due to the high casuistry and impact on animal survival. The evaluation of lymph nodes is part of the staging of mammary tumors and is an important indicator of prognosis. The objective of this study is to highlight the importance of ultrassononografica assessment of axillary and inguinal lymph
\end{abstract}

KEYWORDS: canine, tumour, lymph nodes, metastasis

\section{INTRODUÇÃO}

O tumor de mama é o mais comum em cadelas. Constituem aproximadamente $52 \%$ de todos os tumores que afetam as fêmeas desta espécie, sendo que aproximadamente $50 \%$ são malignos (DE NARDI et al., 2009; FILHO et al., 2010). Tem maior incidência em fêmeas, apenas $1 \%$ de relatos em machos, 
geralmente acomete cadelas não castradas ou castradas tardiamente e geriátricas, é raro em animais com menos de sete anos (LANA et al., 2007).

Segundo SORENMO (2003) a maioria dos tumores mamários são malignos, caracterizados por apresentar um crescimento rápido, aderência aos tecidos adjacentes, ulceração e envolvimento nos gânglios linfáticos. Estas neoplasias podem metastizar para os linfonodos, pulmões e fígado.

O sistema linfático é considerado a principal rota de metástase de neoplasia mamária. Sendo recomendada a avaliação dos linfonodos como parte do estadiamento, em todas as cadelas com neoplasia mamária, para ajudar no estabelecimento do prognóstico e da melhor conduta terapêutica a ser seguida (SOUZA et al., 2013).

Em estudo realizado por KARAYANNOPOULOU et al. (2005) em cadelas verificaram que $85,7 \%$ das cadelas com metástase nos linfonodos têm o tempo de sobrevida inferior a dois anos. Outra pesquisa desenvolvida em humanos por BERTAGNOLLI et al. (2011) indicou que apenas $20 \%$ a $30 \%$ das pacientes sem mestástase em linfonodo axilar desenvolverão recidivas no prazo de 10 anos, em comparação com aproximadamente $70 \%$ das pacientes com envolvimento do linfonodo axilar.

Existem várias técnicas de imagem para avaliação dos linfonodos, como a cintilografia, tomografia e cintilografia, mas o alto custo limita o uso na rotina da clínica de pequenos animais (TOABI et al., 2004). A ultrassonografia é um método de diagnóstico por imagem disponível, não invasivo, dinâmico, de baixo custo que fornece informações sobre a arquitetura dos órgãos, identificando com precisão condições fisiológicas dos tecidos, bem como suas alterações (NYMAN \& O'BRIEN, 2007; PEIXOTO et al., 2010). Por meio deste exame é possível avaliar as margens, ecotextura, ecogenicidade, tamanho e vascularização dos linfonodos (BASTAN et al., 2009).

O linfonodo axilar localiza-se na face medial da porção distal do músculo redondo maior, anteriormente à artéria e veia toracodorsal. Geralmente o linfonodo axilar é único e varia de 0,5 a $5 \mathrm{~cm}$. Os linfonodos inguinais estão situados $3 \mathrm{~cm}$ cranialmente ao osso púbico na face dorsolateral das glândulas mamárias, podendo variar de 0,5 a $2 \mathrm{~cm}$ (FOSSUM et al., 2008). A imagem ultrassonográfica normal do linfonodo é caracterizada pela identificação de uma estrutura de formato ovalado ou achatado, predominantemente hipoecóica, homogênea e com contornos hiperecóicos bem definidos (CARVALHO, 2004).

\section{RELATO DE CASO}

Foi atendida no Hospital Veterinário da Escola de Medicina Veterinária e Zootecnia da Universidade Federal de Goiás-UFG, uma cadela da raça pit bull, idade 10 anos, com queixa de presença de uma massa na mama. Esta foi encaminhada para o Setor de Oncologia e durante a anamnese a tutora relatou que a evolução da massa foi rápida, não era castrada, já havia cruzado, cio irregular e não fazia uso de anticoncepcional.

No exame físico verificou-se a presença de uma única massa na mama inguinal esquerda, era móvel, maior que $5 \mathrm{~cm}$ e ulcerada. $O$ linfonodo inguinal esquerdo estava aumentado à palpação e o axilar não reacional a palpação.

A fim de pesquisar metástase foram solicitadas radiografias de tórax em três projeções (latero-lateral direita e esquerda e ventro dorsal) e ultrassonografia abdominal, que não revelaram nenhuma alteração. 
Para a realização do exame ultrassonográfico dos linfonodos, a paciente foi posicionada em decúbito dorsal para a realização do exame e utilizado um transdutor linear e convexo multifrequencial $(7,5$ a $12 \mathrm{MHz})$ e ganho de $58 \%$, acoplado a equipamento de ultrassom My Lab TM 30 Vet, (The Esaote Group, Genova, Italy). Foram feitas varreduras e cortes transversais e longitudinais de acordo com CARVALHO (2004).

No exame ultrassonográfico do linfonodo inguinal visibilizou-se arquitetura preservada, contorno irregular, borda definida, forma ovalada, ecogenicidade mista, ecotextura grosseira, vascularização acentuada e mista, características estas sugestivas de metástase (Figura 1).

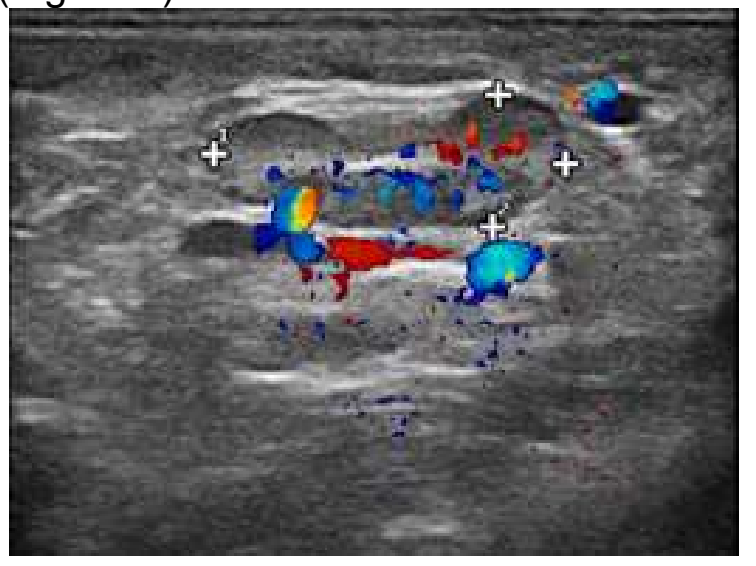

FIGURA 1: Imagem ultrassonográfica em modo Doppler do linfonodo inguinal sugestivo de metástase, demonstrando arquitetura preservada, contorno irregular, borda definida, forma ovalada, ecogenicidade mista, ecotextura grosseira, vascularização acentuada e mista.

O linfonodo axilar apresentou contorno regular, borda definida, arquitetura e ecotextura preservada, ecogenicidade normal e vascularização discreta e hilar, não sugestivo de metástase (Figura 2).

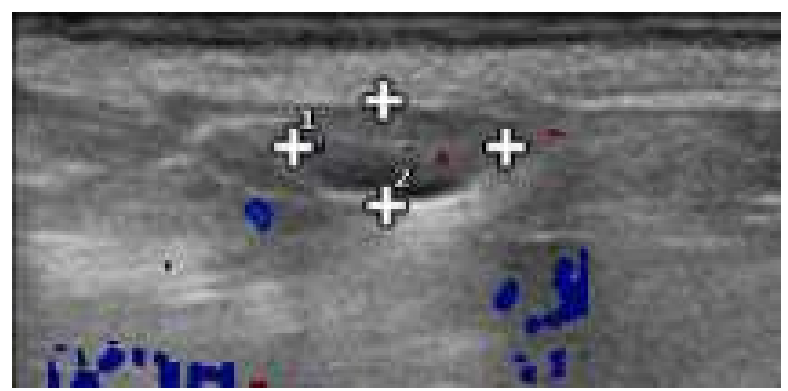

FIGURA 2: Imagem ultrassonográfica em modo Doppler do linfonodo axilar não sugestivo de metástase, demonstrando contorno regular, borda definida, arquitetura e ecotextura preservada, ecogenicidade normal e vascularização discreta e hilar 
Realizou-se mastectomia unilateral e linfadenectomia axilar e inguinal. 0 tumor mamário e linfonodos foram encaminhados para exame histopatológico, onde foram processados conforme a rotina do laboratório da anatomia e patologia e coradas pelo método de Hematoxilina Eosina (HE), nos quais confirmaram a presença de metástase no linfonodo inguinal e classificou-se o tumor como carcinoma mamário padrão tubular grau II de acordo com MISDROP et al. (1999). O linfonodo axilar estava livre de metástase.

Diante do diagnóstico foi instituído tratamento quimioterápico seguindo as orientações de DE NARDI et al.( 2009).

\section{DISCUSSÃO}

A cadela deste relato tinha mais de 7 anos e não era castrada, corroborando com os autores que relatam que tumores de mama acometem com maior frequência fêmeas inteiras ou que foram submetidas tardiamente a ovário-histerectomia (LANA et al., 2007; RIBAS et al., 2012). O tumor era único e estava localizado na mama inguinal, segundo LANA et al., (2007) geralmente 75\% das neoplasias acometem as mamas abdominal caudal (M4) e inguinal (M5), provavelmente por terem mais volume de tecido glandular que as demais.

A ulceração é outra característica relevante na observação e descrição deste tumor mamário. QUEIROGA \& LOPES (2002), ao analisarem histologicamente 57 tumores mamários em cadelas (36 malignos e 21 benignos), observaram que dez dos animais com neoformações malignas apresentaram ulceração na região tumoral ao exame clínico, característica não observada em nenhum dos animais com tumores benignos. O tamanho do tumor também pode ser um indicativo de malignidade, segundo SORENMO (2003) cadelas com tumores com menos de três centímetros de diâmetro estão associados com prognóstico significativamente melhor do que aqueles com mais que três centímetros.

O linfonodo sentinela (LNS) é definido como o primeiro linfonodo a drenar uma neoplasia, e por esta razão será o primeiro sitio a receber a metástase, se ocorrer disseminação linfática, por isso a avaliação dos linfonodos regionais deve sempre fazer parte do estadiamento, e examinados quanto ao tamanho e consistência (ANGELIM \& COELHO, 2012).

Sabe-se que as glândulas torácicas craniais e caudais drenam principalmente para os linfonodos axilares ipsilaterais. A glândula abdominal cranial drena em direção ao linfonodo axilar, porém, também drena para o linfonodo inguinal superficial ipsilateral. Já as glândulas abdominais caudais e inguinais drenam para o linfonodo inguinal superficial ipsilateral. Mas estudos realizados por PATSIKAS et al. (2006) demonstraram que este padrão não se repete em todos os animais, em alguns houve a descrição de conexões linfáticas entre glândulas mamárias adjacentes neoplásicas e normais.

Neste relato, apenas o linfonodo inguinal apresentou aumento de tamanho à palpação. Provavelmente devido a localização do tumor (M5) e a drenagem linfática, que disseminaram as células neoplásicas para 0 linfonodo inguinal. As características ultrassonográficas visibilizadas no linfonodo inguinal como contorno irregular, ecogenicidade mista, ecotextura grosseira, vascularização acentuada condizem com CARVALHO (2004); CHAMMAS et al., (2004). Estes autores descrevem que linfonodos metastáticos tendem a apresentar tais características, as quais foram confirmadas pelo exame histopatológico. 
NYMAN et al. (2005) relatam que a infiltração de células neoplásicas acarreta irregularidades no contorno do linfonodo, e que a ecotextura heterogênea é devido a presença de áreas císticas, decorrentes de hemorragias e necrose tecidual.

Em estudo realizado por MURAMATO et al. (2011) avaliando ultrassonograficamente linfonodos inguinais foi verificado que diante de uma sugestão diagnóstica de processo não neoplásico pode existir uma chance mínima de não o ser, isto ocorre porque falsos negativos foram de apenas $2,2 \%$

\section{CONCLUSÃO}

O exame ultrassonográfico dos linfonodos axilar e inguinal é recomendado para estadiamento de cadelas com neoplasia mamária, por ser um exame acessível e confiável na detecção de metástases.

\section{REFERÊNCIAS}

ANGELIM, J. L.; COELHO, M. C. O. C. Linfonodo sentinela: perspectivas no diagnóstico de metástase no câncer de mama em cadelas: revisão. Medicina Veterinária, Recife, v. 6, n. 1, p. 24-32, 2012.

BASTAN, A.; ÖZENÇ, E.; PIR YAGCI, I. Ultrasonographic evaluation of mammary tumors in bitche. Kafkas Universsitesi Veyeriner Fakultesi Dergisi, v. 1, n. 15, p. 81-86, 2009.

BERTAGNOLLI, A.C.; ESTRELA-LIMA, A.; ALESSI, A.C.; DALECK,C.R.; BUTTRAGO, F.; UEMURA, G.; SENA, M. C. F. Fatores prognósticos em câncer de mama. Comunicacão em Ciencia da Saúde, v. 22, p. 69-81, 2011.

CARVALHO, C. F. Ultra-sonografia em linfonodos. In: CARVALHO, C. F. Ultrasonografia em Pequenos Animais. 1 ed. São Paulo: Roca, 2004, cap. 13, p. 175179

CHAMMAS, M. C., SAITO, O. C; JULIANO, A. G; MARCELINO, A. S. Z; CERRI G.G. 2004. Linfonodos cervicais: Um dilema para o ultrassonografista. Radiol Bras, v. 37, n. 5 , p. 357-364, 2004

DE NARDI, A. B.; RODASKI, S.; ROCHA, N. S.; FERNANDES, S. C. Neoplasias mamárias. In: DALECK, C. R.; DE NARDI, A. B.; RODASKI, S. Oncologia em cães e gatos. 1 ed. São Paulo: Roca, cap. 25, p. 372-380.2009.

FILHO, J. C. O.; KOMMERS, G. D.; MASUDA, E. K.; MARQUES, B. M. F. P.; FIGHERA, R. A.; IRIGOYEN, L. F.; BARROS, C. S. L. Estudo retrospectivo de 1.647 tumores mamários em cães. Pesquisa Veterinária Brasileira, Rio de Janeiro, v.30, n.2, p.177-185, 2010.

FOSSUM, T.W.; HEDLUND, C. S.; JOHNSON, A. L.; SCHULZ, K. S.; SEIM, H. B.; WILLARD, M. D.; BAHR, A.; CARROLL, G. L. Cirurgia de pequenos animais. Rio de Janeiro: Elsevier, 2008.1606p.

KARAYANNOPOULOU, M.; KALDRYMIDOU, E.; CONSTANTINIDIS, T. C.; DESSIRIS, A. Histological grading and prognosis in dogs with mammary carcinomas: ENCICLOPÉDIA BIOSFERA, Centro Científico Conhecer - Goiânia, v.13 n.23; p. 2132016 
aplications of a human grading method. Journal Comp Pathology, v. 133, 246-252, 2005.

LANA, S. E.; RUTTERMAN, G. R.; WITHROW, S. J. Tumors of the mammary gland. In: WITHROW, S.J.; VAIL, D.M. Small Animal Clinical Oncology. 4 ed. ST. Louis: Saunders Elsevier, p. 619-636, 2007.

MISDROP, W.; ELSE, R.W.; HELLMÉN, E.; LIMSCOMB. T. P. Histological classification of mammary tumors of the dog and the cat. In: World Health Organization international histological classification of tumors of domestic animals 2ed. v.7. Washington, D.C. Armed Forces Institute of Pathology in cooperation with the American Registry of Pathology and the World Health Organization Collaborating Center for Worldwide Reference on Comparative Oncology, 1999.

MURAMATO, C.; STERMAN, F. A.; HAGEN, S. C. F.; PINTO, A. C. B. C.; OLIVEIRA, C. M.; FAUSTINO, M.; TALIB, M. S. F.; TORRES, L. N. Avaliação ultrassonográfica de linfonodos na pesquisa de. metástase ne neoplasia mamária em cadelas. Pesq. Vet. Bras, v. 31, n. 11, p. 1006-1013, 2011.

NYMAN, H. T., KRISTENSEN, A. T., SKOVGAARD, I. M.; MCEVOY, F. J. Characterization of normal and abnormal canine super_icial lymph nodes using grayscale B-mode, color _low mapping, power, and super_icial Doppler ultrasonography: A multivariate study. Vet. Radiol. Ultrasound 46:404-410, 2005.

NYMAN, H. T.; O'BRIEN, R. T. The sonographic evaluation of lymph nodes. Clinical Techniques Small Animal Practice, v.22, p. 128-137, 2007.

PATSIKAS, $\quad$ M. N.; KARAYANNOPOULOU, M.; KALDRYMIDOY, E.; PAPADOPOULOU, P. L.; TZEGAS, S. I. The Lymph Drainage of the Neoplastic Mammary Glands in the Bitch: A Lymphographic Study. Anat. Histol. Embryol, v.35, n.4, p.228-34, 2006.

PEIXOTO, G. C. X.; LIRA, R. A.; ALVES, N. D.; SILVA, A. R. Bases físicas de formação de imagem ultrassonográfica. Acta Veterinária Brasilica, v. 4, n. 1, p. 1524, 2010.

QUEIROGA, F.; LOPES, C. Canine mammary tumours, research on new prognostic factors. Revista Portuguesa de Ciências Veterinárias, Lisboa, v.97, p.119-127, 2002.

RIBAS, C. R.; DORNBUSCH, P. T.; FARIA, M. R.; WOUK, A. F. P. F.; CIRIO, S. M. Alterações clínicas relevantes em cadelas com neoplasias mamárias estadiadas. Archives of Veterinary Science, v. 17, n. 1, p. 60-68, 2012.

SORENMO, K. Canine mammry gland tumors. Vet Clin Small Animal, v. 33, p. 573596, 2003. 
SOUZA, F.W.; VELOSO, M.; DE NARDI, A. B.; HUPPES, R. R.; QUARTERONE, C.; RAPOSO, T. M. M.; KASPER, P. N.; OLIVEIRA, M. T.; GUEDES, R. L. Linfadenectomia laparoscópica em cadela com neoplasia mamária. Ciência Rural, v.43, p.750-3, 2013.

TOABI, M.; AQUINO, S. L.; HARISINGHANI, M. G. Current Concepts in Lymph Node Imaging. Journal of Nuclear Medicine, v. 45, n. 9, p. 1509-1518, 2004. 